Bull. Korean Math. Soc. 48 (2011), No. 6, pp. 1329-1339

http://dx.doi.org/10.4134/BKMS.2011.48.6.1329

\title{
OBSTRUCTION FOR THE EXISTENCE OF A PARALLEL VOLUME FORM
}

\author{
Hee-Kyung Cho, Daeyong Kim, and Hyuk Kim*
}

\begin{abstract}
For a manifold with a linear connection, we find an obstruction class to have a volume form parallel with respect to its connection which corresponds to the Chern-Simons secondary invariant in the frame bundle of the manifold.
\end{abstract}

\section{Introduction}

Let $P \in I^{k}(G)$ be an invariant polynomial of a Lie group $G$, namely a symmetric multilinear map $P: \underbrace{\mathfrak{g} \times \cdots \times \mathfrak{g}}_{k \text {-times }} \rightarrow \mathbb{R}$ which is invariant under the adjoint action of $G$. Then given a connection $\omega \in A^{1}(E ; \mathfrak{g})$ on a principal $G$-bundle $\pi: E \rightarrow M$ over a manifold $M$, we can define its curvature $\Omega \in$ $A^{2}(E ; \mathfrak{g})$ as $\Omega=d \omega+\frac{1}{2}[\omega, \omega]$ and by composing $\Omega^{k}$ with $P$, we obtain a $2 k$ form $P\left(\Omega^{k}\right)$ on $E$. Chern and Simons constructed an explicit $(2 k-1)$-form $T P(\omega)$, called now as the Chern-Simons form, such that $d T P(\omega)=P\left(\Omega^{k}\right)$. In the case of $E=O(M)$, the orthonormal frame bundle over $M$, they showed that as a secondary class, namely when $P\left(\Omega^{k}\right)=0,[T P(\omega)] \in H^{2 k-1}(E, \mathbb{R})$ is a conformal invariant ([1]). In the case $k=1, P:=\operatorname{tr}$ and $E=F(M)$, the linear frame bundle over $M$, for a given connection $\omega$ on $F(M)$, we find a class $[\tau] \in H^{1}(M, \mathbb{R})$ as a secondary class on $M$ when $\operatorname{tr} \Omega=0$, satisfying $\left[\pi^{*} \tau\right]=[\operatorname{tr} \omega]=[T P(\omega)] \in H^{1}(E, \mathbb{R})$. This class $[\tau]$ can be in fact described geometrically as an obstruction for the existence of a parallel volume form with respect to $\omega$.

For an orientable manifold with an affine connection, an obstruction class in Čech cohomology for the existence of a volume form parallel with respect to the connection was first obtained in [2]. The class is found in an analytic view point by solving a differential equation globally that turns out to be equivalent to parallelism of a volume form. To understand the class in a geometric view

Received September 8, 2010.

2010 Mathematics Subject Classification. 53B05, 53C05.

Key words and phrases. parallel volume form, parallel volume obstruction class.

* This work was supported by the Korea Research Foundation(KRF) grant funded by the Korea government(MEST)(No. 2010-0016629). 
point, we find the corresponding class in the de Rham cohomology in several different aspects and then also in the simplicial cohomology for the case of a flat affine manifold.

This paper is organized as follows. In Section 2, we observe that to have a volume form parallel with respect to a given connection is equivalent to solving a differential equation involving the connection form considered in [2]. In Section 3, we find the de Rham cohomology class corresponding to the Čech cohomology obstruction class. In Section 4, we show the obstruction class corresponds to the Chern-Simons secondary class and find a relation between it and the holonomy group of the linear frame bundle. In Section 5, we interprete the de Rham cohomology obstruction class in the setting of affine differential geometry. In fact we describe the obstruction to the existence of a volume form parallel with respect to a given connection using a 1-form known as Koszul 1-form ([6]), and show that this Koszul 1-form corresponds our de Rham obstruction class. In Section 6, we find a simplicial cohomology class corresponding to our class especially in the flat affine manifold case, and show that this corresponds to the well known class in the theory of affinely flat manifolds.

\section{Obstruction for a parallel volume form}

For an orientable $n$-manifold $M, F(M)$ can be considered as a principal fibre bundle $E=P(M, G)$ with structure group $G:=G L(n, \mathbb{R})$. Now for $E$, we choose an open covering $\left\{U_{\alpha}\right\}$ of $M$ and get a local trivialization $\psi_{\alpha}$ : $\pi^{-1}\left(U_{\alpha}\right) \rightarrow U_{\alpha} \times G$ defined by $u \mapsto\left(\pi(u), \varphi_{\alpha}(u)\right)$ for a map $\varphi_{\alpha}: \pi^{-1}\left(U_{\alpha}\right) \rightarrow$ $G, \varphi_{\alpha}(u a)=\varphi_{\alpha}(u) a$ for $a \in G$, each $\alpha$. Then we can define transition functions $\psi_{\alpha \beta}: U_{\alpha} \cap U_{\beta} \rightarrow G$ defined by $x \mapsto \varphi_{\alpha}(u)\left(\varphi_{\beta}(u)\right)^{-1}, \pi(u)=x$ such that for a section $\sigma_{\alpha}$ on $U_{\alpha}$ defined by $\sigma_{\alpha}(x):=\psi_{\alpha}^{-1}(x, e)$, where $e$ is the identity of $G, \sigma_{\beta}(x)=\sigma_{\alpha}(x) \psi_{\alpha \beta}(x)$ (see [5, p. 51]). In fact, we can put $\sigma_{\alpha}=\left(\frac{\partial}{\partial x_{\alpha}^{1}}, \ldots, \frac{\partial}{\partial x_{\alpha}^{n}}\right), \sigma_{\beta}=\left(\frac{\partial}{\partial x_{\beta}^{1}}, \ldots, \frac{\partial}{\partial x_{\beta}^{n}}\right)$ for local charts $\left(U_{\alpha}, x_{\alpha}\right),\left(U_{\beta}, x_{\beta}\right)$ and then since $\sigma_{\beta}=\sigma_{\alpha}\left(\frac{\partial x_{\alpha}}{\partial x_{\beta}}\right)_{n \times n}, \psi_{\alpha \beta}=\left(\frac{\partial x_{\alpha}}{\partial x_{\beta}}\right)_{n \times n}=: A_{\alpha \beta}$. A connection in $F(M)$ is called a linear connection of $M$. Let $\nabla$ be a linear connection on $M$. Denote the connection 1 -form of $\nabla$ by $\omega$. Define a $\mathfrak{g}$-valued 1 -form $\omega_{\alpha}$ on $U_{\alpha}$ by $\omega_{\alpha}=\sigma_{\alpha}^{*} \omega$. For $x \in U_{\alpha} \cap U_{\beta}$, since $\sigma_{\beta}(x)=\sigma_{\alpha}(x) A_{\alpha \beta}(x)$, we can get $\omega_{\beta}=A d_{\left(A_{\alpha \beta}\right)^{-1} \omega_{\alpha}+\left(A_{\alpha \beta}\right)^{-1} d A_{\alpha \beta} \text { and hence }}$

$$
\operatorname{tr} \omega_{\beta}=\operatorname{tr} \omega_{\alpha}+d \log \left(\operatorname{det}\left(A_{\alpha \beta}\right)\right) .
$$

Now we consider an equation which is related to the existence of $\nabla$-parallel volume form on $M$.

Proposition 2.1. There exists a local solution $G_{\alpha}$ on $U_{\alpha}$ of an equation

$$
d G=\operatorname{tr} \omega_{\alpha}
$$

if and only if $\mu=\exp \left(G_{\alpha}\right) d x_{\alpha}^{1} \wedge d x_{\alpha}^{2} \wedge \cdots \wedge d x_{\alpha}^{n}$ is $\nabla$-parallel volume form on $U_{\alpha}$. 
Proof. Take a local chart $\left\{x_{\alpha}^{1}, \ldots, x_{\alpha}^{n}\right\}$ on $U_{\alpha}$ and put $X_{i}=\frac{\partial}{\partial x_{\alpha}^{i}}$ for $i=$ $1,2, \ldots, n$. Then $\mu=\exp \left(G_{\alpha}\right) d x_{\alpha}^{1} \wedge d x_{\alpha}^{2} \wedge \cdots \wedge d x_{\alpha}^{n}$ on $U_{\alpha}$ for some function $G_{\alpha}$. Thus

$$
\begin{aligned}
\left(\nabla_{X} \mu\right)\left(X_{1}, \ldots, X_{n}\right) & =\nabla_{X}\left(\mu\left(X_{1}, \ldots, X_{n}\right)\right)-\sum_{i} \mu\left(X_{1}, \ldots, \nabla_{X} X_{i}, \ldots, X_{n}\right) \\
& =\nabla_{X} \exp \left(G_{\alpha}\right)-\sum_{i} \mu\left(X_{1}, \ldots, \sum_{j} \omega_{\alpha}^{j}(X) X_{j}, \ldots, X_{n}\right) \\
& =X\left(\exp \left(G_{\alpha}\right)\right)-\sum_{i} \omega_{\alpha i}^{i}(X) \exp \left(G_{\alpha}\right) \\
& =d\left(\exp \left(G_{\alpha}\right)\right)(X)-\operatorname{tr}\left(\omega_{\alpha}(X)\right) \exp \left(G_{\alpha}\right) \\
& =d G_{\alpha}(X) \exp \left(G_{\alpha}\right)-\operatorname{tr}\left(\omega_{\alpha}(X)\right) \exp \left(G_{\alpha}\right) \\
& =\left(d G_{\alpha}(X)-\operatorname{tr}\left(\omega_{\alpha}(X)\right)\right) \exp \left(G_{\alpha}\right) \\
& =\left(d G_{\alpha}-\operatorname{tr}\left(\omega_{\alpha}\right)\right)(X) \exp \left(G_{\alpha}\right) .
\end{aligned}
$$

A necessary and sufficient condition to solve the equation (2) locally is $\operatorname{tr} \Omega=$ 0 since $\operatorname{tr} \Omega=\operatorname{tr}(d \omega)=d \operatorname{tr} \omega$, where $\Omega$ is the curvature form of $\nabla$. Under the assumption that $\operatorname{tr} \Omega=0$, an obstruction class for the global solvability of the equation (2) in terms of the Čech cohomology is considered in [2]. The equation is solved globally if and only if the obstruction class vanishes. From now on, we call it the " $\nabla$-parallel volume obstruction class" or simply "parallel volume obstruction class" if the given connection $\nabla$ is well understood. Choose a local solution $G_{\alpha}$ of $d G=\operatorname{tr} \omega_{\alpha}$ on each $U_{\alpha}$. Then on $U_{\alpha} \cap U_{\beta}, G_{\beta}-G_{\alpha}-$ $\log \left(\operatorname{det}\left(A_{\alpha \beta}\right)\right)$ is constant by (1). We denote this constant by $c_{\alpha \beta}$ as in [2]. Then $\left[\left\{c_{\alpha \beta}\right\}\right]$ is the parallel volume obstruction class in $\check{H}^{1}(M ; \mathbb{R})$.

\section{Obstruction class in the de Rham cohomology}

Given a linear connection $\nabla$ on $M$ with $\operatorname{tr} \Omega=0$, take a partition of unity $\left\{f_{\alpha}\right\}$ subordinate to $\left\{U_{\alpha}\right\}$ on $M$. Consider a 1 -form

$$
\left.\tau\right|_{U_{\alpha}}=\operatorname{tr} \omega_{\alpha}+\sum_{U_{\alpha} \cap U_{\gamma} \neq \emptyset} d\left(f_{\gamma} \log \operatorname{det}\left(A_{\alpha \gamma}\right)\right) \quad \text { on } U_{\alpha}
$$

On $U_{\alpha} \cap U_{\beta}$,

$$
\begin{aligned}
\left.\tau\right|_{U_{\alpha}}-\left.\tau\right|_{U_{\beta}}= & \left\{\operatorname{tr} \omega_{\alpha}+\sum_{U_{\alpha} \cap U_{\gamma} \neq \emptyset} d\left(f_{\gamma} \log \operatorname{det}\left(A_{\alpha \gamma}\right)\right)\right\} \\
& -\left\{\operatorname{tr} \omega_{\beta}+\sum_{U_{\beta} \cap U_{\gamma} \neq \emptyset} d\left(f_{\gamma} \log \operatorname{det}\left(A_{\beta \gamma}\right)\right)\right\} \\
= & \operatorname{tr} \omega_{\alpha}-\operatorname{tr} \omega_{\beta}+\sum_{U_{\alpha} \cap U_{\beta} \cap U_{\gamma} \neq \emptyset} d\left(f_{\gamma}\left(\log \operatorname{det}\left(A_{\alpha \gamma}\right)-\log \operatorname{det}\left(A_{\beta \gamma}\right)\right)\right)
\end{aligned}
$$




$$
\begin{aligned}
& =\operatorname{tr} \omega_{\alpha}-\operatorname{tr} \omega_{\beta}+\sum_{U_{\alpha} \cap U_{\beta} \cap U_{\gamma} \neq \emptyset} d\left(f_{\gamma} \log \operatorname{det}\left(A_{\alpha \beta}\right)\right) \\
& =\operatorname{tr} \omega_{\alpha}-\operatorname{tr} \omega_{\beta}+d\left(\log \operatorname{det}\left(A_{\alpha \beta}\right)\right)=0 \quad \text { by }(1) .
\end{aligned}
$$

Hence $\tau$ is a well-defined 1-form on $M$. And since $d \tau=d\left(\operatorname{tr} \omega_{\alpha}\right)=d\left(\operatorname{tr}\left(\sigma_{\alpha}^{*} \omega\right)\right)=$ $\sigma_{\alpha}^{*}(d(\operatorname{tr} \omega))=\sigma_{\alpha}^{*}(\operatorname{tr} \Omega)=0,[\tau] \in H_{\mathrm{deRham}}^{1}(M ; \mathbb{R})$.

Proposition 3.1. $[\tau] \in H_{\mathrm{deRham}}^{1}(M ; \mathbb{R})$ is independent of the choice of the partition of unity.

Proof. Take another partition of unity $\left\{f_{\alpha}^{\prime}\right\}$ and define $\tau^{\prime}$ as above. Then

$$
\begin{aligned}
\left.\left(\tau-\tau^{\prime}\right)\right|_{U_{\alpha}}= & \left\{\operatorname{tr} \omega_{\alpha}+\sum_{U_{\alpha} \cap U_{\gamma} \neq \emptyset} d\left(f_{\gamma} \log \operatorname{det}\left(A_{\alpha \gamma}\right)\right)\right\} \\
& -\left\{\operatorname{tr} \omega_{\alpha}+\sum_{U_{\alpha} \cap U_{\gamma} \neq \emptyset} d\left(f_{\gamma}^{\prime} \log \operatorname{det}\left(A_{\alpha \gamma}\right)\right)\right\} \\
= & \sum_{U_{\alpha} \cap U_{\gamma} \neq \emptyset} d\left(\left(f_{\gamma}-f_{\gamma}^{\prime}\right) \log \operatorname{det}\left(A_{\alpha \gamma}\right)\right) \\
= & d\left(\sum_{U_{\alpha} \cap U_{\gamma} \neq \emptyset}\left(f_{\gamma}-f_{\gamma}^{\prime}\right) \log \operatorname{det}\left(A_{\alpha \gamma}\right)\right) .
\end{aligned}
$$

To complete the proof, it is sufficient to show that

$$
\sum_{U_{\alpha} \cap U_{\gamma} \neq \emptyset}\left(f_{\gamma}-f_{\gamma}^{\prime}\right) \log \operatorname{det}\left(A_{\alpha \gamma}\right)
$$

is in fact a well-defined global function. On $U_{\alpha} \cap U_{\beta}$, we have

$$
\begin{aligned}
& \sum_{U_{\alpha} \cap U_{\gamma} \neq \emptyset}\left(f_{\gamma}-f_{\gamma}^{\prime}\right) \log \operatorname{det}\left(A_{\alpha \gamma}\right) \\
= & \sum_{U_{\alpha} \cap U_{\beta} \cap U_{\gamma} \neq \emptyset}\left(f_{\gamma}-f_{\gamma}^{\prime}\right) \log \operatorname{det}\left(A_{\alpha \gamma}\right) \\
= & \left.\sum_{U_{\alpha} \cap U_{\beta} \cap U_{\gamma} \neq \emptyset}\left(f_{\gamma}-f_{\gamma}^{\prime}\right) \log \operatorname{det}\left(A_{\alpha \beta}\right)+\log \operatorname{det}\left(A_{\beta \gamma}\right)\right) \\
= & \sum_{U_{\alpha} \cap U_{\beta} \cap U_{\gamma} \neq \emptyset}\left(f_{\gamma}-f_{\gamma}^{\prime}\right) \log \operatorname{det}\left(A_{\alpha \beta}\right)+\sum_{U_{\beta} \cap U_{\gamma} \neq \emptyset}\left(f_{\gamma}-f_{\gamma}^{\prime}\right) \log \operatorname{det}\left(A_{\beta \gamma}\right) \\
= & \log \operatorname{det}\left(A_{\alpha \beta}\right)\left(\sum_{U_{\alpha} \cap U_{\beta} \cap U_{\gamma} \neq \emptyset} f_{\gamma}-\sum_{U_{\alpha} \cap U_{\beta} \cap U_{\gamma} \neq \emptyset} f_{\gamma}^{\prime}\right) \\
& +\sum_{U_{\beta} \cap U_{\gamma} \neq \emptyset}\left(f_{\gamma}-f_{\gamma}^{\prime}\right) \log \operatorname{det}\left(A_{\beta \gamma}\right) \\
= & \sum_{U_{\beta} \cap U_{\gamma} \neq \emptyset}\left(f_{\gamma}-f_{\gamma}^{\prime}\right) \log \operatorname{det}\left(A_{\beta \gamma}\right) .
\end{aligned}
$$

Hence $[\tau]=\left[\tau^{\prime}\right]$. 
Theorem 3.2. $[\tau] \in H_{\mathrm{deRham}}^{1}(M ; \mathbb{R})$ is the parallel volume obstruction class.

Proof. Since $\left.d \tau\right|_{U_{\alpha}}=d\left(\operatorname{tr} \omega_{\alpha}\right)=0$, there exists $G_{\alpha}$ on $U_{\alpha}$ such that $\operatorname{tr} \omega_{\alpha}=$ $d G_{\alpha}$. Then

$$
\begin{gathered}
\left.\tau\right|_{U_{\alpha}}=d\left(G_{\alpha}+\sum_{U_{\alpha} \cap U_{\gamma} \neq \emptyset} f_{\gamma} \log \operatorname{det}\left(A_{\alpha \gamma}\right)\right), \\
\left.\tau\right|_{U_{\beta}}=d\left(G_{\beta}+\sum_{U_{\beta} \cap U_{\gamma} \neq \emptyset} f_{\gamma} \log \operatorname{det}\left(A_{\beta \gamma}\right)\right), \\
\left(G_{\beta}+\sum_{U_{\beta} \cap U_{\gamma} \neq \emptyset} f_{\gamma} \log \operatorname{det}\left(A_{\beta \gamma}\right)\right)-\left(G_{\alpha}+\sum_{U_{\alpha} \cap U_{\gamma} \neq \emptyset} f_{\gamma} \log \operatorname{det}\left(A_{\alpha \gamma}\right)\right) \\
=G_{\beta}-G_{\alpha}-\log \operatorname{det}\left(A_{\alpha \beta}\right)=c_{\alpha \beta} .
\end{gathered}
$$

So $[\tau] \in H_{\text {deRham }}^{1}(M ; \mathbb{R})$ corresponds to $\left[\left\{c_{\alpha \beta}\right\}\right] \in \check{H}(M ; \mathbb{R})$ by the de Rham isomorphism ([4, p. 226]).

When $[\tau]$ is trivial, i.e., $\tau=d g$ for some function $g$ on $M$, consider the volume form

$$
d V=\exp \left(g-\sum_{U_{\alpha} \cap U_{\gamma} \neq \emptyset} f_{\gamma} \log \operatorname{det}\left(A_{\alpha \gamma}\right)\right) d x_{\alpha}^{1} \wedge \cdots \wedge d x_{\alpha}^{n} .
$$

It is easy to show that this volume form is a well-defined global form by similar computation as before. Also note that $d\left(g-\sum_{U_{\alpha} \cap U_{\gamma} \neq \emptyset} f_{\gamma} \log \operatorname{det}\left(A_{\alpha \gamma}\right)\right)=\operatorname{tr} \omega_{\alpha}$ clearly holds by the definition of $\tau$. Hence it is parallel with respect to $\nabla$ by Proposition 2.1.

\section{A secondary class and a relation with the holonomy group}

Proposition 4.1. $\pi^{*}\left(\operatorname{tr} \omega_{\alpha}\right)+d \log \left|\operatorname{det} \varphi_{\alpha}\right|=\operatorname{tr} \omega$ on $\pi^{-1}\left(U_{\alpha}\right)$.

Proof. By (1), since

$$
\begin{aligned}
& \pi^{*}\left(\operatorname{tr} \omega_{\beta}\right)=\pi^{*}\left(\operatorname{tr} \omega_{\alpha}\right)+\pi^{*} d \log \left|\operatorname{det}\left(A_{\alpha \beta}\right)\right|=\pi^{*}\left(\operatorname{tr} \omega_{\alpha}\right)+d \pi^{*}\left(\log \left|\operatorname{det}\left(A_{\alpha \beta}\right)\right|\right) \\
&=\pi^{*}\left(\operatorname{tr} \omega_{\alpha}\right)+d \log \left|\operatorname{det}\left(A_{\alpha \beta} \circ \pi\right)\right|=\pi^{*}\left(\operatorname{tr} \omega_{\alpha}\right)+d \log \left|\operatorname{det}\left(\varphi_{\alpha} \varphi_{\beta}^{-1}\right)\right| \\
&=\pi^{*}\left(\operatorname{tr} \omega_{\alpha}\right)+d \log \left|\operatorname{det} \varphi_{\alpha} \operatorname{det}\left(\varphi_{\beta}^{-1}\right)\right|=\pi^{*}\left(\operatorname{tr} \omega_{\alpha}\right)+d \log \left|\frac{\operatorname{det} \varphi_{\alpha}}{\operatorname{det} \varphi_{\beta}}\right| \\
&=\pi^{*}\left(\operatorname{tr} \omega_{\alpha}\right)+d \log \left|\operatorname{det} \varphi_{\alpha}\right|-d \log \left|\operatorname{det} \varphi_{\beta}\right|, \\
& \pi^{*}\left(\operatorname{tr} \omega_{\beta}\right)+d \log \left|\operatorname{det} \varphi_{\beta}\right|=\pi^{*}\left(\operatorname{tr} \omega_{\alpha}\right)+d \log \left|\operatorname{det} \varphi_{\alpha}\right| \text { on } \pi^{-1}\left(U_{\alpha}\right) \cap \pi^{-1}\left(U_{\beta}\right) .
\end{aligned}
$$
Thus a 1-form on the principal bundle $F(M)$ which is locally expressed by

$$
\pi^{*}\left(\operatorname{tr} \omega_{\alpha}\right)+d \log \left|\operatorname{det} \varphi_{\alpha}\right| \quad \text { on } \pi^{-1}\left(U_{\alpha}\right)
$$


is a well-defined global 1-form on $F(M)$. Now let $X=h X+v X$ be any vector field on $F(M)$, where $h X$ and $v X$ are the horizontal part and the vertical part of $X$ respectively. Then for $u \in F(M)$,

$$
\begin{aligned}
& \left(\pi^{*}\left(\operatorname{tr} \omega_{\alpha}\right)+d \log \left|\operatorname{det} \varphi_{\alpha}\right|\right)\left(X_{u}\right) \\
= & \left(\operatorname{tr}\left(\left(\sigma_{\alpha} \circ \pi\right)^{*} \omega\right)+d \log \left|\operatorname{det} \varphi_{\alpha}\right|\right)\left(X_{u}\right) \\
= & \operatorname{tr}\left(\omega\left(\left(\sigma_{\alpha} \circ \pi\right)_{*} X_{u}\right)\right)+\operatorname{tr}\left(\left(\varphi_{\alpha}(u)\right)^{-1}\left(\varphi_{\alpha *} X_{u}\right)\right) \\
(3)= & \operatorname{tr}\left(\omega\left(\left(v\left(\left(\sigma_{\alpha} \circ \pi\right)_{*} X\right)\right)_{\sigma_{\alpha(x)}}\right)\right)+\operatorname{tr}\left(\left(\varphi_{\alpha}(u)\right)^{-1}\left(\varphi_{\alpha *}\left((h X)_{u}+(v X)_{u}\right)\right) .\right.
\end{aligned}
$$

On the one hand, since $\pi=\pi \circ\left(\sigma_{\alpha} \circ \pi\right), h X=h\left(\left(\sigma_{\alpha} \circ \pi\right)_{*} X\right)$ and so

$$
\varphi_{\alpha *}\left((h X)_{u}\right)=\varphi_{\alpha *}\left(\left(h\left(\left(\sigma_{\alpha} \circ \pi\right)_{*} X\right)_{\sigma_{\alpha}(x)}\right) .\right.
$$

And since $\left(\varphi_{\alpha} \circ \sigma_{\alpha} \circ \pi\right)(u)=e, \varphi_{\alpha *}\left(\left(\sigma_{\alpha} \circ \pi\right)_{*} X\right)=0$ and then

$$
\varphi_{\alpha *}\left(\left(h\left(\left(\sigma_{\alpha} \circ \pi\right)_{*} X\right)_{\sigma_{\alpha}(x)}\right)=-\varphi_{\alpha *}\left(\left(v\left(\left(\sigma_{\alpha} \circ \pi\right)_{*} X\right)_{\sigma_{\alpha}(x)}\right) .\right.\right.
$$

On the other hand,

$$
\left(\varphi_{\alpha}(u)\right)^{-1}\left(\varphi_{\alpha *}(v X)_{u}\right)=\varphi_{\alpha *}(v X)_{\sigma_{\alpha(x)}}=\omega\left(\left(\varphi_{\alpha *}(v X)_{\sigma_{\alpha(x)}}\right)_{u}^{*}\right)=\omega\left((v X)_{u}\right) .
$$

Then (3) equals to

$$
\begin{aligned}
& \operatorname{tr}\left(\omega\left(\left(v\left(\left(\sigma_{\alpha} \circ \pi\right)_{*} X\right)\right)_{\sigma_{\alpha(x)}}\right)\right)+\operatorname{tr}\left(\left(\varphi_{\alpha}\left(\sigma_{\alpha}(x)\right)\right)^{-1} \varphi_{\alpha *}\left(\left(h\left(\left(\sigma_{\alpha} \circ \pi\right)_{*} X\right)_{\sigma_{\alpha}(x)}\right)\right)\right. \\
& +\operatorname{tr}\left(\left(\varphi_{\alpha}(u)\right)^{-1} \varphi_{\alpha_{*}}\left((v X)_{u}\right)\right) \\
= & \operatorname{tr}\left(\omega\left(\left(v\left(\left(\sigma_{\alpha} \circ \pi\right)_{*} X\right)\right)_{\sigma_{\alpha(x)}}\right)\right)-\operatorname{tr}\left(\left(\varphi_{\alpha}\left(\sigma_{\alpha}(x)\right)\right)^{-1} \varphi_{\alpha_{*}}\left(\left(v\left(\left(\sigma_{\alpha} \circ \pi\right)_{*} X\right)_{\sigma_{\alpha}(x)}\right)\right)\right. \\
& +\operatorname{tr}\left(\omega\left((v X)_{u}\right)\right) \\
= & \operatorname{tr}\left(\omega\left(\left(v\left(\left(\sigma_{\alpha} \circ \pi\right)_{*} X\right)\right)_{\sigma_{\alpha(x)}}\right)\right)-\operatorname{tr}\left(\omega\left(\left(v\left(\left(\sigma_{\alpha} \circ \pi\right)_{*} X\right)\right)_{\sigma_{\alpha(x)}}\right)\right)+\operatorname{tr}\left(\omega\left(X_{u}\right)\right) \\
= & \operatorname{tr}\left(\omega\left(X_{u}\right)\right) .
\end{aligned}
$$

Proposition 4.2. $\left[\pi^{*} \tau\right]=[\operatorname{tr} \omega]$.

Proof. On $\pi^{-1}\left(U_{\alpha}\right)$,

$$
\begin{aligned}
\pi^{*} \tau-\operatorname{tr} \omega & =\pi^{*}(\tau)-\left(\pi^{*}\left(\operatorname{tr} \omega_{\alpha}\right)+d \log \left|\operatorname{det} \varphi_{\alpha}\right|\right) \\
& =\sum_{U_{\alpha} \cap U_{\gamma} \neq \emptyset} \pi^{*} d\left(f_{\gamma} \log \operatorname{det}\left(A_{\alpha \gamma}\right)\right)-d \log \left|\operatorname{det} \varphi_{\alpha}\right| \\
& =d\left(\sum_{U_{\alpha} \cap U_{\gamma} \neq \emptyset} \pi^{*}\left(f_{\gamma} \log \operatorname{det}\left(A_{\alpha \gamma}\right)\right)-\log \left|\operatorname{det} \varphi_{\alpha}\right|\right) .
\end{aligned}
$$

Now it is sufficient to show that $\sum_{U_{\alpha} \cap U_{\gamma} \neq \emptyset} \pi^{*}\left(f_{\gamma} \log \operatorname{det}\left(A_{\alpha \gamma}\right)\right)-\log \left|\operatorname{det} \varphi_{\alpha}\right|$ is a well-defined global function on $E$. On $\pi^{-1}\left(U_{\alpha}\right) \cap \pi^{-1}\left(U_{\beta}\right)$,

$$
\begin{aligned}
& \left\{\sum_{U_{\alpha} \cap U_{\gamma} \neq \emptyset} \pi^{*}\left(f_{\gamma} \log \operatorname{det}\left(A_{\alpha \gamma}\right)\right)-\log \left|\operatorname{det} \varphi_{\alpha}\right|\right\} \\
& -\left\{\sum_{U_{\beta} \cap U_{\gamma} \neq \emptyset} \pi^{*}\left(f_{\gamma} \log \operatorname{det}\left(A_{\beta \gamma}\right)\right)-\log \left|\operatorname{det} \varphi_{\beta}\right|\right\}
\end{aligned}
$$




$$
\begin{aligned}
& =\sum_{U_{\alpha} \cap U_{\beta} \cap U_{\gamma} \neq \emptyset} \pi^{*}\left(f_{\gamma} \log \operatorname{det}\left(A_{\alpha \beta}\right)\right)-\left(\log \left|\operatorname{det} \varphi_{\alpha}\right|+\log \left|\operatorname{det} \varphi_{\beta}^{-1}\right|\right) \\
& =\pi^{*}\left(\log \operatorname{det}\left(A_{\alpha \beta}\right)\right)-\log \left|\operatorname{det}\left(\varphi_{\alpha} \varphi_{\beta}^{-1}\right)\right| \\
& =\log \operatorname{det}\left(A_{\alpha \beta} \circ \pi\right)-\log \operatorname{det}\left(A_{\alpha \beta} \circ \pi\right)=0 .
\end{aligned}
$$

Now by computing the volume obstruction, we can get the following result.

Theorem 4.3. $[\tau]=0$ if and only if the linear holonomy group is contained in $S L(n, \mathbb{R})$.

Proof. For a point $u$ in $E$, take a loop $\rho$ in $M$ based at $\pi(u)$. Then there exists a unique horizontal lift $\rho^{*}$ of $\rho$ which starts from $u$. And

$$
\begin{aligned}
\int_{\rho^{*}} \operatorname{tr} \omega= & \int_{\rho^{*}} \sum_{\alpha}\left\{\operatorname{tr}\left(\pi^{*} \omega_{\alpha}\right)+d\left(\log \left|\operatorname{det} \varphi_{\alpha}\right|\right)\right\} \\
= & \sum_{\alpha} \int_{\rho^{*}} \pi^{*}\left(\operatorname{tr}\left(\omega_{\alpha}\right)\right)+\sum_{\alpha} \int_{\rho^{*}} d\left(\log \left|\operatorname{det}\left(\varphi_{\alpha}\right)\right|\right) \\
= & \sum_{\alpha} \int_{\rho} \operatorname{tr}\left(\omega_{\alpha}\right)+\log \left|\operatorname{det}\left(\varphi_{\alpha}\left(\rho^{*}(1)\right)\right)\right|-\log \left|\operatorname{det}\left(\varphi_{\alpha}\left(\rho^{*}(0)\right)\right)\right| \\
= & \sum_{\alpha}\left(\int_{\rho} \operatorname{tr}\left(\omega_{\alpha}\right)+\sum_{U_{\alpha} \cap U_{\beta} \neq \emptyset} \int_{\rho} d\left(f_{\beta} \log \left|\operatorname{det}\left(\psi_{\alpha \beta}\right)\right|\right)\right) \\
& +\log \left|\operatorname{det}\left(\varphi_{\alpha}(u g)\right)\right|-\log \left|\operatorname{det}\left(\varphi_{\alpha}(u)\right)\right| \\
= & \int_{\rho} \sum_{\alpha}\left\{\operatorname{tr}\left(\omega_{\alpha}\right)+\sum_{U_{\alpha} \cap U_{\beta} \neq \emptyset} \int_{\rho} d\left(f_{\beta} \log \left|\operatorname{det}\left(\psi_{\alpha \beta}\right)\right|\right)\right\} \\
& +\log \left|\operatorname{det}\left(\varphi_{\alpha}(u) g\right)\right|-\log \left|\operatorname{det}\left(\varphi_{\alpha}(u)\right)\right| \\
= & \int_{\rho} \tau+\log \left|\operatorname{det}\left(\varphi_{\alpha}(u)\right) \operatorname{det} g\right|-\log \left|\operatorname{det}\left(\varphi_{\alpha}(u)\right)\right| \\
= & \int_{\rho} \tau+\log |\operatorname{det} g|
\end{aligned}
$$

where $g$ is the element in the holonomy group corresponding to $\rho$. However, since $\omega=0$ on $\rho^{*}, 0=\int_{\rho} \tau+\log |\operatorname{det} g|$ and thus $\int_{\rho} \tau=-\log |\operatorname{det} g|$. Hence $[\tau]=0$ if and only if $|\operatorname{det} g|=1$.

Corollary 4.4. A connection $\nabla$ has a parallel volume form if and only if $\operatorname{tr} \Omega=0$ and the linear holonomy group of $\nabla$ is contained in $S L(n, \mathbb{R})$.

\section{Parallel volume obstruction class using Koszul 1-form}

For a linear connection $\nabla$ with $\operatorname{tr} \Omega=0$ given on an orientable $n$-dimensional manifold $M$, let $\mu$ be a volume form. Define a 1-form $\theta_{\mu}$ as $\nabla_{X} \mu=\theta_{\mu}(X) \mu$ for all $X \in \Gamma(T M)$. Take a local chart $\left\{x^{1}, \ldots, x^{n}\right\}$ and let $\mu=\exp (G) d x^{1} \wedge$ $d x^{2} \wedge \cdots \wedge d x^{n}$ and put $X_{i}=\frac{\partial}{\partial x^{i}}$ for $i=1,2, \ldots, n$. Abusing the notation, let 
$\omega$ and $\Omega$ be the corresponding connection and curvature forms. Then as in the proof of Proposition 2.1,

$$
\left(\nabla_{X} \mu\right)\left(X_{1}, \ldots, X_{n}\right)=\{d G(X)-\operatorname{tr}(\omega(X))\} \exp (G) .
$$

On the other hand,

$$
\left(\theta_{\mu}(X) \mu\right)\left(X_{1}, \ldots, X_{n}\right)=\theta_{\mu}(X) \mu\left(X_{1}, \ldots, X_{n}\right)=\theta(X) \exp (G) .
$$

Thus locally,

$$
\theta_{\mu}=d G-\operatorname{tr} \omega .
$$

And since $d \theta_{\mu}=d(d G-\operatorname{tr} \omega)=-d \operatorname{tr} \omega=-\operatorname{tr} \Omega=0,\left[\theta_{\mu}\right] \in H_{\text {deRham }}^{1}(M ; \mathbb{R})$. For another volume form $\nu=\exp (F) \mu$,

$$
\begin{aligned}
\nabla_{X} \nu=\nabla_{X}(\exp (F) \mu) & =d(\exp (F))(X) \mu+\exp (F) \theta_{\mu}(X) \mu \\
& =\theta_{\nu}(X) \nu=\theta_{\nu}(X) \exp (F) \mu .
\end{aligned}
$$

Then $\theta_{\nu}=\theta_{\mu}+\exp (-F) d \exp (F)=\theta_{\mu}+d F$ and thus $\left[\theta_{\mu}\right]=\left[\theta_{\nu}\right]$. Hence for any connection $\nabla$ with $\operatorname{tr} \Omega=0, \theta_{\nabla}:=\left[\theta_{\mu}\right]$ for some volume form $\mu$ on $M$ is well-defined and we can get easily the following result equivalent to the one obtained in [2].

Theorem 5.1. For a linear connection $\nabla$ with $\operatorname{tr} \Omega=0$ given on an orientable $n$-dimensional manifold $M, \theta_{\nabla}=0$ if and only if there exists a $\nabla$-parallel volume form. In this case, such parallel form is unique up to constant.

Proof. $(\Rightarrow) \theta_{\nabla}=\left[\theta_{\mu}\right]=0$ for some volume form $\mu$ on $M$. Then $\theta_{\mu}=d F$ for some function $F$. Now define $\nu=\exp (-F) \mu$ and we show that this form is $\nabla$-parallel.

$$
\begin{aligned}
\nabla_{X} \nu=\nabla_{X}(\exp (-F) \mu) & =d(\exp (-F))(X) \mu+\exp (-F) \nabla_{X} \mu \\
& =-\exp (-F) d F(X) \mu+\exp (-F) d F(X) \mu \\
& =0 \quad \text { for all } X \in \Gamma(T M) .
\end{aligned}
$$

$(\Leftarrow)$ Let $\mu$ be a $\nabla$-parallel volume form. Then $\theta_{\mu}=0$ and hence $\theta_{\nabla}=\left[\theta_{\mu}\right]=0$. If $\mu^{\prime}=\exp \left(F^{\prime}\right) \mu$ is another parallel volume form, then we have $0=\theta_{\mu^{\prime}}=$ $\theta_{\mu}+d F^{\prime}=d F^{\prime}$. Hence such form is unique up to constant.

Proposition 5.2. For a linear connection $\nabla$ with $\operatorname{tr} \Omega=0$ given on an orientable $n$-dimensional manifold $M, \theta_{\nabla} \in H^{1}(M ; \mathbb{R})$ is the $\nabla$-parallel volume obstruction class.

Proof. In fact,

$$
\begin{aligned}
\left.\tau\right|_{U_{\alpha}} & =\operatorname{tr} \omega_{\alpha}+\sum_{U_{\alpha} \cap U_{\gamma} \neq \emptyset} d\left(f_{\gamma} \log \operatorname{det}\left(A_{\alpha \gamma}\right)\right) \\
& =\operatorname{tr} \omega_{\alpha}-d G_{\alpha}+d G_{\alpha}+\sum_{U_{\alpha} \cap U_{\gamma} \neq \emptyset} d\left(f_{\gamma} \log \operatorname{det}\left(A_{\alpha \gamma}\right)\right)
\end{aligned}
$$




$$
=-\theta_{\mu_{\alpha}}+d\left(\sum_{U_{\alpha} \cap U_{\gamma} \neq \emptyset} f_{\gamma}\left(G_{\alpha}+\log \operatorname{det}\left(A_{\alpha \gamma}\right)\right)\right)
$$

where $\mu_{\alpha}=\exp \left(G_{\alpha}\right) d x_{\alpha}^{1} \wedge \cdots \wedge d x_{\alpha}^{n}$ on $U_{\alpha}$. Then

$$
\begin{aligned}
\left.\tau\right|_{U_{\alpha}}+\theta_{\mu_{\alpha}} & =d\left(\sum_{U_{\alpha} \cap U_{\gamma} \neq \emptyset} f_{\gamma}\left(G_{\alpha}+\log \operatorname{det}\left(A_{\alpha \gamma}\right)\right)\right) \\
& =d\left(\sum_{U_{\alpha} \cap U_{\gamma} \neq \emptyset} f_{\gamma}\left(G_{\alpha}+\log \operatorname{det}\left(A_{\alpha \gamma}\right)+c_{\alpha \gamma}\right)\right) .
\end{aligned}
$$

Recall $c_{\alpha \beta}=G_{\beta}-G_{\alpha}-\log \operatorname{det} A_{\alpha \beta}$ is a constant. And on $U_{\alpha} \cap U_{\beta}$, since

$$
\begin{aligned}
& \sum_{U_{\alpha} \cap U_{\beta} \cap U_{\gamma} \neq \emptyset} f_{\gamma}\left(G_{\beta}+\log \operatorname{det}\left(A_{\beta \gamma}\right)+c_{\beta \gamma_{0}}\right) \\
& -\sum_{U_{\alpha} \cap U_{\beta} \cap U_{\gamma} \neq \emptyset} f_{\gamma}\left(G_{\alpha}+\log \operatorname{det}\left(A_{\alpha \gamma}\right)+c_{\alpha \gamma_{0}}\right) \\
= & \sum_{U_{\alpha} \cap U_{\beta} \cap U_{\gamma} \neq \emptyset} f_{\gamma}\left(G_{\beta}-G_{\alpha}-\log \operatorname{det}\left(A_{\alpha \gamma}\right)-\log \operatorname{det}\left(A_{\gamma \beta}\right)+c_{\beta \gamma_{0}}-c_{\alpha \gamma_{0}}\right) \\
= & \sum_{U_{\alpha} \cap U_{\beta} \cap U_{\gamma} \neq \emptyset} f_{\gamma}\left(G_{\beta}-G_{\alpha}-\log \operatorname{det}\left(A_{\alpha \beta}\right)+c_{\beta \gamma_{0}}+c_{\gamma_{0} \alpha}\right) \\
= & \sum_{U_{\alpha} \cap U_{\beta} \cap U_{\gamma} \neq \emptyset} f_{\gamma}\left(c_{\alpha \beta}+c_{\beta \gamma_{0}}+c_{\gamma_{0} \alpha}\right)=0,
\end{aligned}
$$

$\sum_{U_{\alpha} \cap U_{\gamma} \neq \emptyset} f_{\gamma}\left(G_{\alpha}+\log \operatorname{det}\left(A_{\alpha \gamma}\right)+c_{\alpha \gamma_{0}}\right)$ is a global function. Notice that $c_{\alpha \beta}=$ $-c_{\beta \alpha}$ and $c_{\alpha \beta}+c_{\beta \gamma}+c_{\gamma \alpha}=0$ hold from its definition. And thus $\left[\tau+\theta_{\mu}\right]=0$ and hence $[\tau]=\left[-\theta_{\mu}\right]=-\theta_{\nabla}$.

\section{Parallel volume obstruction class on affine flat manifold}

Let $M$ be a $n$-dimensional manifold with a flat affine structure. $D$ is a developing map from $\tilde{M}$, the universal covering of $M$, into $\mathbb{R}^{n}$. A triangulation $K$ of $M$ can be lifted to a triangulation $\tilde{K}$ of $\tilde{M}$. Take a dual complex of $K$ (See [3, pp. 80-81]). For each dual 1-simplex $(a b)$ of $M$, we choose the developing image of a lifting $(\tilde{a b})$ of $(a b)$. Then the image is contained in the union of two $n$-simplices $\triangle_{\tilde{a}}$ and $\triangle_{\tilde{b}}$ with common side such that $\triangle_{\tilde{a}}$ and $\triangle_{\tilde{b}}$ are the developing images of liftings of each $n$-simplices $a$ and $b$. Under an affine transformation, the ratio of the volumes of two $n$-simplices is preserved. So we take a map $\phi$ defined by $(a b) \mapsto \log \left(\frac{\operatorname{vol}\left(\triangle_{\tilde{a}}\right)}{\operatorname{vol}\left(\triangle_{\tilde{b}}\right)}\right)$. Since

$$
\phi((b a))=\log \left(\frac{\operatorname{vol}\left(\triangle_{\tilde{b}}\right)}{\operatorname{vol}\left(\triangle_{\tilde{a}}\right)}\right)=-\phi((a b)),
$$

$\phi$ is a 1-cochain in $M$. 
Let $\left(a_{0}, \ldots, a_{k}\right)$ be a dual 2-simplex which has 1-faces $\left(a_{0} a_{1}\right),\left(a_{1} a_{2}\right), \ldots$, $\left(a_{k} a_{0}\right)$. Since $\left(a_{0} a_{1}\right)+\left(a_{1} a_{2}\right)+\cdots+\left(a_{k} a_{0}\right)$ is a trivial loop in $M$, the developing image of its lifting $\left(\widetilde{a_{0} a_{1}}\right)+\left(\widetilde{a_{1} a_{2}}\right)+\cdots+\left(\widetilde{a_{k} a_{0}}\right)$ is also a loop.

$$
\begin{aligned}
\delta \phi\left(\left(a_{0}, a_{1}, \ldots, a_{k}\right)\right) & =\phi\left(\left(a_{0} a_{1}\right)+\left(a_{1} a_{2}\right)+\cdots+\left(a_{k} a_{0}\right)\right) \\
& =\log \left(\frac{\operatorname{vol}\left(\triangle_{\tilde{a}_{0}}\right)}{\operatorname{vol}\left(\triangle_{\tilde{a}_{1}}\right)}\right)+\log \left(\frac{\operatorname{vol}\left(\triangle_{\tilde{a}_{1}}\right)}{\operatorname{vol}\left(\triangle_{\tilde{a}_{2}}\right)}\right)+\cdots+\log \left(\frac{\operatorname{vol}\left(\triangle_{\tilde{a}_{k}}\right)}{\operatorname{vol}\left(\triangle_{\tilde{a}_{0}}\right)}\right) \\
& =0 .
\end{aligned}
$$

Hence, $\phi$ is a 1-cocycle.

Theorem 6.1. $[\phi] \in H^{1}(M ; \mathbb{R})$ is the parallel volume obstruction class.

Proof. Let $\rho=\left(x_{0} x_{1}\right)+\left(x_{1} x_{2}\right)+\cdots+\left(x_{m} x_{0}\right)$. Then a lifting of $\rho$ is $\left(\widetilde{x_{0} x_{1}}\right)+$ $\left(\widetilde{x_{1} x_{2}}\right)+\cdots+\left(\widetilde{x_{m} x_{m+1}}\right)$.

$$
\begin{aligned}
\phi(\rho) & =\log \left(\frac{\operatorname{vol}\left(\triangle_{\tilde{x}_{0}}\right)}{\operatorname{vol}\left(\triangle_{\tilde{x}_{1}}\right)}\right)+\log \left(\frac{\operatorname{vol}\left(\triangle_{\tilde{x}_{1}}\right)}{\operatorname{vol}\left(\triangle_{\tilde{x}_{2}}\right)}\right)+\cdots+\log \left(\frac{\operatorname{vol}\left(\triangle_{\tilde{x}_{m}}\right)}{\operatorname{vol}\left(\triangle_{\tilde{x}_{m+1}}\right)}\right) \\
& =\log \left(\operatorname{vol}\left(\triangle_{\tilde{x}_{0}}\right)\right)-\log \left(\operatorname{vol}\left(\triangle_{\tilde{x}_{m+1}}\right)\right) .
\end{aligned}
$$

$\triangle_{\tilde{x}_{m+1}}$ is the image of $\triangle_{\tilde{x}_{0}}$ by $g$ in the holonomy group corresponding to $\rho$. So $\operatorname{vol}\left(\triangle_{\tilde{x}_{m+1}}\right)$ is equal to $|\operatorname{det} g| \operatorname{vol}\left(\triangle_{\tilde{x}_{0}}\right)$. Hence

$$
\phi(\rho)=-\log |\operatorname{det} g| \text {. }
$$

Now the proof follows from the computation in the proof of Theorem 4.3.

Remark 6.2. Using linear holonomy, we obtain a homomorphism of $\pi_{1}(M)$ to $G L(n, \mathbb{R})$. Consider the composition of this homomorphism with $-\log |\operatorname{det}|$ : $\pi_{1}(M) \rightarrow G L(n, \mathbb{R}) \rightarrow \mathbb{R}$. Since $\mathbb{R}$ is abelian, this map induces a map from $H_{1}(M)$ into $\mathbb{R}$. Then it is an element in $H^{1}(M ; \mathbb{R})$, and is exactly same as $\phi$ by Theorem 6.1 . When $[\phi]$ is trivial, the volume is determined up to constant multiple by the volume of the developing image of a lifting. As an example, consider a $\mathbb{Z} \times \mathbb{Z}=\langle\alpha, \beta\rangle$ action on the real plane $\mathbb{R} \times \mathbb{R}$ defined by $\alpha(x, y)=$ $(x+s t y+1, y+s)$ and $\beta(x, y)=(x+t y, y+1)$ for any real number $s$ and $t$. Then it gives a flat structure for the same torus. Indeed $\alpha$ is represented by $\left(\begin{array}{lll}1 & \text { st } & 1 \\ 0 & 1 & s \\ 0 & 0 & 1\end{array}\right)$ and $\beta$ by $\left(\begin{array}{lll}1 & t & 0 \\ 0 & 1 & 1 \\ 0 & 0 & 1\end{array}\right)$ as an element of $\operatorname{Aff}(2, \mathbb{R})$. So the linear holonomy group is contained in $S L(n, \mathbb{R})$. By Theorem 4.3, the volume obstruction is trivial. Actually, $d x \wedge d y$ is a parallel volume form. Take $u$ and $v$ in $\mathbb{C}$ such that $|u|,|v|>1$, and $u^{m} \neq v^{n}$ for any $m, n \in \mathbb{N}$. Let $\mathbb{Z} \times \mathbb{Z}=\langle\gamma, \delta\rangle$ acts on $\mathbb{C}$ by $\gamma(z)=u z$ and $\delta(z)=v z$. Then it gives a flat structure for the affine torus. The element in the holonomy group corresponding to $\gamma$ and $\delta$ respectively is $\left(\begin{array}{cc}u_{1} & -u_{2} \\ u_{2} & u_{1}\end{array}\right)$ and $\left(\begin{array}{cc}v_{1} & -v_{2} \\ v_{2} & v_{1}\end{array}\right)$ as a real linear map, where $u=u_{1}+i u_{2}$ and $v=v_{1}+i v_{2}$. Their determinants are $|u|^{2}$ and $|v|^{2}$ which cannot be equal to 1 . So the volume obstruction is not trivial and hence this structure doesn't have a parallel volume form. 
Remark 6.3. Let $\nabla_{0}$ be a metric connection coming from a metric $g_{0}$ over $M$. Then any connection $\nabla$ on $M$ has a form $\nabla_{0}+\alpha$ for some $\alpha \in \Gamma\left(T^{*} M \otimes\right.$ $\left.T^{*} M \otimes T M\right)$. And $\Omega=d \omega+\omega \wedge \omega=\Omega_{0}+d \alpha+\alpha \wedge \alpha+\omega \wedge \alpha+\alpha \wedge \omega$. Thus $\left\{\nabla=\nabla_{0}+\alpha \mid \operatorname{tr} \Omega=0\right\} \cong\left\{\alpha \in \Gamma\left(T^{*} M \otimes T^{*} M \otimes T M\right) \mid \operatorname{tr} \alpha\right.$ is closed $\}=: \mathcal{S}$ is a vector space. Now define $\theta: \mathcal{S} \rightarrow H^{1}(M, \mathbb{R})$ by $\alpha \mapsto[\theta(\alpha)]=[-\operatorname{tr} \alpha]=\theta_{\nabla}$. Then $\theta$ is linear and $\mathcal{K}:=\operatorname{ker} \theta=\{\alpha \mid \operatorname{tr} \alpha$ is exact $\}$. Moreover we have $\mathcal{M} \subset \mathcal{K} \subset \mathcal{S}$ for the set $\mathcal{M}$ of metric connections over $M$. Note that $\theta$ is onto since if $\beta$ is a closed 1 -form on $M$, then $\beta=\operatorname{tr} \alpha$ for $\alpha:=\frac{1}{n} \beta I_{n}$. Hence $\mathcal{S} / \mathcal{K} \cong H^{1}(M, \mathbb{R})$.

\section{References}

[1] S. S. Chern and J. Simons, Characteristic forms and geometric invariants, Ann. of Math. (2) 99 (1974), 48-69.

[2] D. P. Chi and Y. D. Yoon, On an affine connection which admits a volume-like form, Canad. Math. Bull. 33 (1990), no. 4, 482-488.

[3] B. A. Dubrovin, A. T. Fomenko, and S. P. Novikov, Modern Geometry - Methods and Applications, Part III, Springer-Verlag, 1990.

[4] W. Greub, S. Halperin, and R. Vanstone, Connections, Curvature and Cohomology. Vol. I: De Rham cohomology of manifolds and vector bundles, Academic Press, 1972.

[5] S. Kobayashi and K. Nomizu, Foundations of Differential Geometry, Vol I, Interscience Publishers, 1963.

[6] H. Shima, On certain locally flat homogeneous manifolds of solvable Lie groups, Osaka J. Math. 13 (1976), no. 2, 213-229.

[7] W. P. Thurston, Geometry and topology of 3-manifolds. Lecture Notes, Princeton University, 1979.

HeE-Kyung Cho

120-603 Hyundai Apt.

Apgujeong-Dong, Gangnam-Gu

SeOul 135-110, Korea

DAEYONG KIM

Department of Mathematical Sciences

Seoul National University

SEOUL 151-747, KorEA

E-mail address: dykim@math.snu.ac.kr

HYUK KIM

Department of Mathematical Sciences

Seoul National University

SEOUl 151-747, Korea

E-mail address: hyukkim@snu.ac.kr 